\title{
Le régime fiscal de la livraison gratuite des biens liés à la maladie de covid-19 par les entreprises industrielles et commerciales en R.D. Congo.
}

\author{
Par Jean-Victor MBOYO EMPENGE ea IKONA*
}

\section{Résumé}

Cet article examine la question de la libre livraison des biens corporels liée au régime fiscal de la covid-19 dans un contexte d'analyser relationnelle entre la TVA et de l'impôt sur le Bénéfice professionnel et Profits des sociétés commerciales face aux activités de ces entreprises qui doivent participer à la solidarité nationale par la livraison gratuite de biens corporels liés au covid-19 aux tiers-bénéficiaires. Ainsi, considérée comme une livraison pour soi-même, elle obligerait l'entreprise concernée à déclarer et reverser la TVA collectée, au respect de mécanisme de déductibilité. L'administration fiscale qui se veut gardien des recettes fiscales doit participer à l'action du gouvernement en sécurisant les entreprises, qui manifestent l'intention de participer à l'effort de la solidarité nationale de lutte contre le Covid-19, face au risque de droit de reprise des impositions au moyen des redressements fiscaux, pour absence du garantie fiscale du contribuable vérifié.

Aider son voisin, est une maxime qui n'est pas au goût du fisc, surtout lorsqu'elle est pratiquée par une entreprise dont la mission est l'exploitation de l'objet social et non d'exercer la charité. Ladite opération pourrait-elle être exonérée au taux zéro sur la TVA à l'intérieur des biens liés au covid-19. Pour cette raison, l'administration fiscale doit garantir le contribuable contre tout changement interprétative ultérieure sur la question liée à la livraison gratuite des biens liés au covid-19, à un prix inférieur au prix de revient, à des tiersbénéficiaires autre que l'organisme public.

\begin{abstract}
This article examines the question relative to the free delivery of tangibles goods linked to covid-19 tax law regime. The context was to analyze the relation between the VAT tax law and company tax law with the industrial and commercial companies activities. Those companies need to participate to the national solidarity against the covid-19. In fact, even if the free delivery of tangible goods linked to covid-19 who'd be considered as a self-delivery assimilated to the delivery for himself, without payment to the company, and oblige normally to pay the VAT tax. This question obliges the tax administration to reform, by the finances law, the tax law according to the company benefice tax and the Vat tax and, help
\end{abstract}

* MBOYO EMPENGE ea IKONA Jean-Victor, Chef de travaux à la Faculté de droit de l'Université de Kinshasa Avocat au Barreau de Kinshasa/Matété, Courriel : jnvicmboyo@gmail.com. 
the companies to participate to the national solidarity against covid-19, and protect the company against the risk of retracement of his account.

The first time is to reform the present VAT tax law by the financial law or the administration ability to create, by the circular or instruction called "tax doctrine", the formal administration position to the legal or fact question, whom the fiscal law didn't give answer. So, "Help his neighbor is a maxim which is not to the taste of the taxman", especially when it is practiced by a company whose mission is to make profit and benefit from its savings, and not charity". Within this legal framework, the free delivery of tangible goods linked to covid-19 would be considered as a self-delivery assimilated to a delivery against payment for the company.

This delivery exposes the company to tax risks of recovery with tax penalties for an abnormal management act even though it's a matter of public health. For this reason, no classic justifications drawn from a commercial or capitalistic relationship are presented. However, the struggle remains a long-term process compelling the Congolese rulers not to fall into the European model of a state of emergency and to take formally accepted fiscal positions by means of administration instructions or circular which clear intelligibility in relation to the situations of global crisis impacting the countries. Furthermore, the tax administration must guarantee the taxpayer against any changement of another subsequent interpretation on legal issue related to free delivery or at a lower price than the cost price of goods related to Covid-19 by companies to third parties because it is exempt from regularization and also from the taxation of self-delivery, and this throughout the period of Covid-19. In this context, Covid-19 products will be cost less than presently, because of the reasons of public utilities, and general and humanitarian interest were been formally accepted by tax doctrine in interpretation of 41 VAT law article as amended to date as non-taxable for selfdelivery. The administration tax doctrine putting tax legality into state, because the first one is omnipotent in the taw administration life's.

\section{Introduction}

Le coronavirus autrement appelé Covid-19 est une abréviation anglaise de « coronavirus disease-2019 ». Elle est une maladie infectieuse émergente qui est causée par une souche de coronavirus appelée SARS-COV-2 et appartient ainsi à une grande famille de virus, les coronavirus. Ces derniers qui doivent leur nom à la forme de couronne qu'ont les protéines qui les enrobent, font partie d'une vaste partie de virus dont certains infectent différents animaux, d'autres d'hommes. Ses symptômes les plus fréquents sont la fièvre, la toux, le gène respiratoire susceptible d'occasionner le décès du patient, avec un taux de mortalité très fortement dépendant de son âge. ${ }^{1}$, est une question de société qui touche aux valeurs de l'Etat de droit que sont celles de démocratie, de liberté et des droits de l’Homme défendues par la

1 Jonas KIBALA KUMA, «L'économie mondiale face à la pandémie de Covid-19 : Etats des lieux, analyses et perspectives ", HAL.archives-ouvertes.fr/hal-02888395, 3 Juillet 2020, Visionconférence_IRES_FASEG_UNIKIN, 11/07/2020,p.5. 
Fondation Konrad. Elle relève de la gouvernance en matière de la "Santé publique »et de l' "économie nationale », les deux réalités étant indissociables, la question de l'économie étant difficile à gérer en cette période particulière de la pandémie ${ }^{2}$.

Dans ce contexte qu'il faut inscrire l'organisation du présent séminaire dont le thème est « l'Etat de droit à l'ère de Covid-19 ». C'est en rapport à cette thématique que notre réflexion entend porter sur le sou-thème « L'avenir de l'économie de la RD Congo au passage de Coronavirus par les entreprises congolaises ». Quatre éléments sont mis en exergue. Il s'agit de « l'Etat de droit, la gestion de la livraison gratuite des biens, le Covid-19, le régime fiscal ».

La préoccupation est de savoir si une entreprise peut bénéficier de l'article 417 de la loi sur la TVA par la livraison à titre gratuit des biens liés à la maladie de Covid-19 pour des raisons de solidarité nationale et de coopération d'avec l'Etat? Le Covid-19 étant une pandémie et un fléau mondial nécessitant la sécurité sanitaire de tous, la tolérance administrative en ce qui concerne la fiscalité en faveur des entreprises qui réalisent de don lié au Covid-19 devrait-elle être pérenne ou une mesure périodique? Qu'en est-il du régime fiscal des biens et services sortis des actifs de l'entreprises et cédés à titre gratuit soit comme dons ou des cadeaux liés au Covid-19 en faveur de n'importe quel bénéficiaire? S'agit-il d'un acte anormal de gestion et ces biens sont-ils exclus de la déductibilité pour fraude à la loi sur la TVA?

Cette étude entend aider l'administration fiscale à réfléchir sur les positions formellement admises susceptibles d'être prises au niveau national et à valider celles qui l'ont déjà été par le gouvernement en les comparants aux initiatives d'autres administrations pour permettre aux entreprises de protéger leur Capital, considérer à juste titre comme l'élément producteur de l'assiette fiscale. Cela doit davantage être envisagé en cette période de la pandémie de Covid-19, période durant laquelle les entreprises estiment, pour des raisons de solidarité participative à la lutte contre le Coronavirus, de recevoir protection fiscale à ces actes de gestion dont le fisc apprécierait le caractère normal ou anormal.

L'intérêt de cette analyse est double, sur le plan de la gouvernance fiscale redevable de la santé publique et de la sauvegarde des entreprises donatrices puis, l'avenir de l'assiette fiscale au Congo, à l'ère de la pandémie sous étude. Et, sur le plan juridique dans le cadre de la protection de l'entreprise participative à la solidarité nationale face au Coronavirus, dont la totipotence fiscale a effets de produire des prises de positions fiscales formellement admise venant en supplétif de la loi inintelligible. Pour asseoir notre hypothèse, qu'une doctrine administrative puise indiquant selon l'article 39 la loi $\mathrm{n}^{\circ}$ 004/2003 du 13 mars 2003 portant réforme des procédures fiscales, telle que modifiée et complétée à ce jours ${ }^{3}$ : "Il ne sera procédé à aucun redressement si la cause de celui-ci résulte d'un différend por-

2 Origine : Ville de Wuhan en Chine centrale (dernier trimestre 2019). En Janvier 2020, l'OMS considère la Covid-19 comme une urgence de santé publique et la requalifie comme une pandémie le 11 mars 2020,Jonas KIBALA KUMA, «L'économie mondiale face à la pandémie de Covid-19: Etats des lieux, analyses et perspectives ", p.5.

3 Jean-Marie MBOKO DJ'ANDIMA, Code Général des Impôts, Kinshasa, 2007, p.335. 
tant sur une interprétation d'une disposition fiscale par le redevable de bonne foi, lorsque cette interprétation était formellement admise par l'Administration des Impôts à l'époque des faits ». Sur ce, l'Administration fiscale peut, par rapport à la question de la livraison gratuite par des entreprises des biens liés au covid-19 à la société pour des besoins de solidarité, formellement prendre une position fiscale sur l'article 41,7 de l'Ordonnance-loi sur la TVA, en indiquant : "la livraison gratuite des biens liés au Covid-19 serait dispensé de la régularisation et par conséquent de la taxation pour livraison à soi même, car opérée pour des raisons d'intérêt général, de sécurité sanitaire et d'utilité publique, comme effort à la solidarité nationale et à la coopération avec le gouvernement.

L'économie de l'article 39 de la loi portant réforme de procédure fiscale précise que l'administration des impôts peut prendre une position de droit en interprétant l'article 41, point 7 de 1'Ordonnance-Loi n ${ }^{\circ}$ 10/011 du 20 Août 2010 portant institution de la Taxe sur la Valeur Ajoutée telle que modifiée à ce jour, dans l'idée que : "N'ouvre pas droit à déduction, la taxe ayant grevé les biens cédés et les services rendus gratuitement ou à un prix inférieur au prix de revient, à titre des dons, cadeaux, ...quelle que soit la qualité du bénéficiaire, sauf quand il s'agit d'objets publicitaires de faible valeur unitaire hors taxe ou de la livraison à titre gratuit ou à un prix inférieur des matériels liés à Covid-19 au tiers».

Comment y arriver? La question « comment » renvoie dit-on à une question méthodologique, de savoir quelles sont les démarches à suivre, sur le plan légal que sociologique, pour ne pas permettre aux entreprises qui veuillent participer au Fond de soutien pour la riposte, la prévention et la détection de covid-19, de ne pas être rappeler par le fisc. Le fait sanitaire de Covid-19 ayant précédé les lois fiscales sur la TVA et l'impôt sur les bénéfices et profits», il y a lieu de pallier à ce vide juridique, il faut primo, de lege ferenda par des réformes fiscales, au moyen des loi de finances, secundo l'interprétation administrative du fisc sur des questions liées à la participation des entreprises à l'effort tant national que mondial relatif à la sauvegarde de la santé publique à l'égard de covid-19.

Pour ce, nos développements vont s'articuler autour de quatre points. Le Contour du régime fiscal de la livraison à titre gratuit à de tiers par les entreprises (A), L'Etude des objectifs comparables de la livraison à titre gratuit des biens liés au covid-19 en droits français, belge et congolais (B), les Regards critiques sur la législation congolaise (C) et enfin Perspectifs d'avenir et propositions (D).

\section{A. Contour du régime fiscal de la livraison à titre gratuit des biens de l'entreprise aux tiers}

\section{Contours définitionnel et conceptuel du Régime fiscal}

La livraison à titre gratuit des biens de l'entreprise à des tiers, organisme public ou privé, est assimilable à la livraison de biens à soi même (LBSM), qui par définition serait lorsqu'un assujetti a, pour une même opération, la double qualité de " fournisseur » et de 
« consommateur final » ${ }^{4}$; consommant à titre personnel ce qui a été loué à l'objet social de son entreprise.

Le principe de neutralité justifie qu'une telle opération soit soumise à la TVA bien qu'elle ne mette pas en présence deux personnes distinctes et qu'il n'y a pas une contrepartie en espèce ou en nature.

Dans ce cadre, la livraison à titre gratuit de biens de l'entreprise à des tiers reste un prélèvement des biens de l'actif du bilan pour usage privé, bien que affectés à un tiers de par la volonté du « consommateur-entreprise ». C'est de l' « autoconsommation » ou de l' « autofabrication » ou de l'achat aux fins d'exploitation mais autoconsommé. En droit fiscal, les livraisons de biens meubles corporels et les prestations de services, à titre onéreux ou à titre gratuit, faites à des tiers, peut importe soit l'objectif, sont assujettis à la taxe sur la valeur ajoutée, sauf pour les cas de livraisons faits à l'Etat et à ses démembrements. L'article 9 du même texte précité sur la TVA, le précise, en l'assimilant à la livraison à soi-même.

Par livraison à soi-même, il faut entendre les prélèvements et affectations effectués par les assujettis pour des besoins d'exploitation, en cas de production d'immobilisations ou de biens exclus du droit à déduction ou encore des besoins autres que ceux de l'exploitation, à savoir ceux des dirigeants, de son personnel ou des tiers $^{5}$.

Dans le cas où il ya livraisons de biens de l'entreprise à des tiers, à titre onéreux ou à titre gratuit, l'opération est assujettie à la TVA et, l'administration ne peut sous aucun prétexte déroger à la loi fiscale, qui se veut d'ordre public de non seulement de contrainte mais aussi de protection.

Pour le cas sous examen, l'entreprise agissant comme «fournisseur et consommateur » de ses biens liés au Covid-19, des biens qui sont livrés aux tiers-bénéficiaires, pour des raisons non professionnelles de solidarité nationale. Cette opération, assimilable à la livraison à soi-même, est imposable à la TVA, en application de mesure de déductibilité, pièces comptables à l'appui de la comptabilité. Nonobstant cela, pour qu'elle puisse bénéficier du régime de l'exonération à la TVA, il faut, de lege ferenda, une disposition légale au moyen de réforme fiscale de l'actuelle loi sur la TVA, comme c'est le cas avec ces biens livrés de covid-19 qui sont déductibles de l'impôt sur les bénéfices et profits; soit une interprétation admise de disposition obscure ou une circulaire interprétative qui ajoute à la loi et, qui sur pied de l'article 39 de la loi sur la procédure fiscale garantie le contribuable de bonne foi contre tout risque de droit de rappel, en cas de changement d'interprétation administrative. Cette disposition est une garantie du contribuable contre l'arbitraire du fisc et le risque de redressement.

4 Maurice COZIAN et Florence DEBOISSY, Précis de fiscalité des entreprises, 2010-2011, 34ème édition, Paris, p. 457.

5 République Démocratique du Congo, Ministère des finances, Code des Impôts, Ordonnance-loi n ${ }^{\circ}$ 10/001 du 20 août 2010 modifié à ce jour, Kinshasa, article 9, 2014, p.172. 


\section{L'Administration fiscale, le Covid-19 et l'entreprise}

La Covid-19 n'est pas qu'une question sanitaire, elle à un effet boule de neige, tant politique, économique, fiscale que socioculturelle. Elle paralyse, à son passage, les droits et libertés civils, économiques et sociaux du « citoyen-contribuable », la gouvernance et la démocratie d'un Etat qui se veut un «Etat de droit », dans sa conception démocratique.

Dans ce cadre, la pandémie anime la pensée de chaque Etat et par ricochet, de chaque Administration, notamment fiscale qui prend part à cet effort de solidarité mondiale, en allégeant les charges sanitaires liées à ladite pandémie.

La locution «face à » place d'emblée l'Entreprise, qui de principe est imposable, en opposition d'une possibilité d'exonération possible, à accorder à la livraison gratuite des biens liés "Coronavirus » à des tiers, en cas de reforme ou d'une circulaire interprétative favorable au contribuable. Une tentative d'aversion-aversion, entre l'impôt à devoir et des raisons de solidarité nationale. Car, le but recherché ou obtenus par l'entreprise en livrant gratuitement ses biens à des tiers, pour des raisons de solidarité à la santé publique, ne peut déductible de l'impôt sur les bénéfices et profits, car elles ne constituent des charges qui ont été engagées pour des raisons d'exploitation de l'entreprise.

Pour permettre aux entreprises, en termes général, d'assister l'Etat, à la participation à l'effort national contre le Covid-19 et ses effets néfastes, il faut une gouvernance fiscale protectrice des entreprises donatrices des biens liés au coronavirus à des tiers bénéficiaires, qu'elles soient protégées soit par une loi de finances reformant la loi sur la TVA, comme c'est le cas avec la loi de finances 2020 qui exonère les dons et contributions liés au covid-19 donnés au Fonds de soutien de lutte contre la pandémie à l'impôt sur les sociétés, soit par une interprétation administrative, comme la directive française. Car, le patrimoine privé, au même titre que les finances publiques, sont sacrées ${ }^{6}$. Tel est l'essence de l'article 61 de la Constitution congolaise qui dispose : « En aucun cas, et même lorsque l'état de siège ou l'état d'urgence aura été proclamé conformément aux articles 85 et 86 de la présente Constitution, il ne peut être dérogé aux droits et principes fondamentaux énumérés ciaprès : 1. Le droit à la vie... " ${ }^{7}$. Pour ce, le "droit à la vie » et la "légalité des impôts » prévue à l'article 174, al.1 de la Constitution de la RD. Congo, aux fins de faciliter l'entreprise industrielle ou commerciale à être exonérée de la livraison gratuite des biens liés au Covid-19 à des tiers.

Ce problème du régime fiscal de l'opération sous étude est apprécié sur le plan politique, en ce sens-ci que l'Etat de droit appel au respect de la légalité. Cette dernière doit être

6 République Démocratique du Congo, Constitutions de la République Démocratique du Congo modifiée par la loi $\mathrm{n}^{\circ} 11 / 002$ du 20 janvier 2011 portant révision de certains articles de la Constitution de la République Démocratique du Congo du 18 février 2006, in JORDC, 52 ème Année, Numéro Spécial, Kinshasa, 5 février 2011, article 34, al. 1.

7 République Démocratique du Congo, Constitutions de la République Démocratique du Congo modifiée par la loi $\mathrm{n}^{\circ} 11 / 002$ du 20 janvier 2011 portant révision de certains articles de la Constitution de la République Démocratique du Congo du 18 février 2006, in JORDC, 52 ème Année, Numéro Spécial, Kinshasa, 5 février 2011, article 34, al. 1. 
en adéquation avec le fait, notamment la question de « droit à la vie et par ricochet à la santé publique ». Sur le plan économique, pour sécuriser juridiquement les économies des entreprises, le législateur doit devoir, comme dit, de lege refenda, prendre des mesures de reforme, soit procéder par une interprétation administrative de l'article 41,7 de la loi sur la TVA, de manière à rende la question sous examen, non imposable. A la TVA à l'intérieur. Ceci permet de pérenniser l'assiette fiscale et protéger les actes de gestion des entreprises quant à ce.

\section{Le Régime fiscal de la livraison d'un bien à titre gratuit aux tiers}

1. Opérations imposables à la TVA par nature

Il est de principe que, la livraison d'un bien à titre gratuit à un même régime fiscal que la sa livraison à titre onéreux des biens de l'entreprise à des personnes différentes et à titre onéreux, telle est l'essence de l'article 9 de la loi sur la TVA ${ }^{8}$.

L'assimilation de la de la livraison à titre gratuit à la livraison à soi-même de biens affectés aux besoins de l'entreprise ${ }^{9}$ pour que l'entreprise ${ }^{10}$ assujettie rend ladite entreprise consommateur, à titre personnel, imposable à la TVA à l'intérieur ${ }^{11}$.

\section{Opérations exonérées à la TVA par nature}

Certaines opérations sont, par nature, exonérées de la TVA, notamment l'exportation des biens qui est exonérée et la livraison des biens à l'intérieur qui sont, notamment, exonérées de la TVA. A l'exemple de « Des dons, legs ou matériels fournis gratuitement à l'Etat, aux provinces, aux entités territoriales décentralisées et aux organismes de droit public $»^{12}$.

Ceci conduit à dire que la livraison à titre gratuit à des tiers de biens liés au Covid-19 n'est pas, en principe, non imposable à la TVA, sauf disposition fiscale expresse, accord préalable fiscal ou une instruction administrative fiscale interprétant l'article 15,13 in fine, sur l'exonération à la TVA et, sur l'article 41,7 relatif à l'exclusion au mécanisme de déduction, de la loi sur la TVA au Congo en indiquant que les biens et services liés au Covid-19 rendus gratuitement ou à un prix inférieur au prix de revient, à titre de cadeaux, de dons, legs, quelle que soit la qualité du bénéficiaire, organismes public ou privé, seraient exonérés de la TVA et ouvrerait droit au mécanisme de la déductibilité.

8 Aubry GILDAS et Cie, Fiscal, $2^{\text {ème }}$ édition, Paris, 2017, p. 819, n 4073.

9 Francis LEFEBVRE, Fiscal, Mémento Pratique, Paris, 2012, p.728, n 47395.

10 Idem, p.729, $\mathrm{n}^{\circ} 47400$.

11 Maurice COZIAN, Précis de fiscalité des entreprises, 34è édition, Paris, 2010, p.388.

12 Article 15, Loi n 004/2003 du 13 mars 2003 portant réforme des procédures fiscales, telle que modifiée et complétée à ce jours. 
Si, la seule tolérance légale à la déductibilité serait : « sauf quand il s'agit d'objets publicitaires de faible valeur unitaire hors taxe $»^{13}$. Dans cet élan des choses, l'administration fiscale peut admettre, dans le jour à venir, soit par voie de réforme législative, au moyen d'une loi de finances, soit apportée par voie d'une instruction administrative ou une circulaire, une "tolérance administrative » ou signé avec le contribuable un accord préalable fiscal avant tout fait générateur, précisant que les entreprises qui participent à l'effort national contre le Covid-19, au moyen des livraisons à titre gratuit des biens achetés ou fabriqués par elles, seront bénéficiaires du mécanisme de déductibilité qui leur accordera, un crédit TVA sur la TVA déclarée et payée en amont.

Ces mesures fiscales à prendre nécessite une réformation par la loi des finances, de l'article 15,13 sur la TVA relatif à l'exonération à la TVA de toute importation des dons, legs ou matériels fournis gratuitement à l'Etat, aux provinces, aux entités territoriales décentralisées et aux organismes de droit public, mais aussi lorsque ces biens achetés ou fabriqués dans l'intention de la revente, sont fournis gratuitement au Fonds de soutien de riposte contre le covid-19 et/ou aux organismes professionnels de santé publics ou privés, pour des raisons de sécurité sanitaire et de coopérer avec l'Etat dans le cadre de la solidarité nationale, à l'ère de la pandémie de coronavirus. Sur la même logique, une circulaire impérative de l'article 41,7 de l'O.L. sur la TVA, pourrait préciser que ces opérations de livraison des matériels de Covid-19 fournis gratuitement, par les entreprises, aux organismes tant publics que privés, qui en sont bénéficiaires, ne peuvent être exclues de mécanisme de déductibilité et de régularisation. Quid des dons et contributions apportés par les entreprises en rapport avec l'impôt sur les bénéfices et profits.

\section{Opérations exonérées à l'impôt sur le Bénéfice des sociétés}

Le contexte international est marqué par la présence de la pandémie covid-19 depuis, l'an 2019, attestant que les effets de cette épidémie mondiale est multidimensionnelle, tant sur les plans économique, fiscale que socioculturelle. Sur ce, les dons, cadeaux des covid-19 livrés gratuitement par les entreprises au Fonds de soutien à la riposte contre l'épidémie mondiale de covid-19, sont par destination de la loi de finances exonérés. Pour cela, il est disposé ce qui suit : "Il est ajouté à l'ordonnance-loi n 69/009 du 10 février 1969 relative aux impôts cédulaires sur les revenus, un article 46 bis libellé comme suit : "Article 46 bis : Les dons et contributions apportés par les contribuables au Fonds de soutien à la riposte contre la pandémie du coronavirus au cours de l'exercice comptable 2020 sont déductibles du résultat imposable à l'impôt professionnel sur les bénéfices et profits, à condition que ces dépenses soient justifient par des pièces comptables $»^{14}$.

13 République Démocratique du Congo, Ministère des Finances, Code des Impôts, Ordonnance-loi n -13/007 du 23 février 2013 modifiant et complétant certaines dispositions de l'ordonnance-loi n ○10/001 de la 20/08/2010 portante institution de la Taxe sur la Valeur Ajoutée, 2014.

14 Loi de finances $n^{\circ}$ 20/020 du 28/12/2020 pour l'exercice 2021, in JORDC, $n^{\circ}$ Spécial, 62 ${ }^{\circ}$ année, $\mathrm{I}^{\circ}$ Partie, Kinshasa, 8 Janvier 2021, p.19. 
Il sied de noter qu'un nouveau régime d'imposition a été instauré sur la question par la loi de finances $n^{\circ} 20 / 020$ du 28/12/2020 pour l'exercice 2021 précise bien que les dons et contributions apportés par les entreprises au Fonds de soutien à la riposte contre la pandémie du coronavirus au cours de l'exercice comptable 2020 sont déductibles du résultat imposable à l'impôt professionnel sur les bénéfices et profits, à condition que ces dépenses soient justifiées par les pièces comptables.

L'article 30 de la loi de finances de 2020 reconnaît aux dépenses effectuées par la société, dans le cadre de livraison à titre gratuit au Fonds de soutien à la riposte contre l'épidemie mondiale de covid-19, le caractère des dépenses déductibles au résultat comptable imposable à l'impôt sur les bénéfices, alors qu'elles ne sont pas liées directement aux charges d'exploitation de l'objet social de l'entreprise en question en revanche, étant apportés au tiers, elles devraient en principe, être non déductibles.

Quant à nous, de lege ferenda, nous estimons que, chaque loi de finances, à venir, devrait apporter une réforme par rapport à la pandémie de covid-19 qui n'est pas une épidémie, telle que dit dans la loi de finances 2020, en son article 30. Ainsi, les dons, legs, contributions, de toute nature des biens liés au covid-19 fabriqués ou achetés à des fins d'exploitation, mais qui par la suite apportés par l'entreprise commerciale ou industrielle non seulement au Fonds de soutien à la riposte de covid-19, mais aussi à tout établissement hospitalier, des organismes et professionnels de santé, des services d'accueils des personnes âgées, handicapées ou vulnérables, à l'Etat et à ses demembrements, au cours de l'exercice comptable déterminé constitueraient des dépenses déductibles au résultat comptable imposable à l'impôt sur les bénéfices et profits qui est de $30 \%$. A condition d'apporter des pièces justificatives identifiant la date, la nature et la quantité des dons apportés, puis la copie d'attestation de délivrance des biens livrés établis par le bénéficiaire, aux fins de permettre la bonne exécution du contrôle fiscal. Par conséquent, la loi sur la TVA devrait s'inspirer de l'article 30 de la loi de finances $n^{\circ}$ 20/020du 28/12/2020 pour l'exercice físcal 2021, acceptant la déductibilité des biens corporels meubles, des dons et contributions livrés gratuitement par les entreprises au Fonds de soutien à la riposte, aux organismes publics et, à tout organisme de santé, aux professionnels de santé que des services d'accueils des personnes âgées ou handicapées contre covid-19, et préciser par une disposition légale que les Biens liés au covid-19 livrés gratuitement à tout organisme public ou privé, pièces comptables et Attestation de délivrance du bénéficiaire à l'appui, sont exonérés de la TVA sur la consommation. 


\section{B. Etudes des Objectifs comparables de la livraison à titre gratuit des biens de covid-19, en droits français, belge et R.D. Congo}

\section{Les mesures d'assouplissements mises en place par le rescrit français ${ }^{15}$.}

De par définition, le rescrit est une prise de position de l'administration fiscale sur une question du contribuable lui permettant d'obtenir du fisc son avis préalable sur le régime fiscal d'une situation fiscale de fait à passer. Elle est soit individuelle, en conséquence notifiée au contribuable, soit générale par conséquent, publiée dans un bulletin des impôts ou un document officiel de service de publication des actes.

Dans l'esprit de cette directive fiscale française, à savoir, le rescrit du 7/4/2020, mis à jour le 13/05/2020, l'administration fiscale française a étendu le champ d'application de la dispense de reversement pour les dons effectuées à compter du 01/03/2020jusqu'au 30 ème jour suivant la fin de la période de l'état d'urgence sanitaire, dons relatifs aux matériels sanitaires liés au Covid-19 au profit des organismes bénéficiaires, les établissements de santé, les services d'accueils de personnes âgées ou handicapées, les professionnels de santé, les services de l'Etat et les collectivités territoriales ${ }^{16}$.

Le gouvernement français signe un accord préalable fiscal de régularisation de mécanisme de déductibilité et d'exonération à la taxe sur la TVA, la livraison à titre gratuit des biens liés au Covid619 à n'importe quel bénéficiaire, sans tenue de l'attestation de délivrance des biens livrés liés au covid-19, seules les informations comptables de la donatrice permettant d'identifier la date, la nature de don, la quantité et le bénéficiaire, suffisent ${ }^{17}$.

\section{Les mesures d'assouplissements mise en place par la Circulaire belge du 20/20/C/46 Concernant les dons de biens à certains établissements et les dons en nature ${ }^{18}$.}

La Belgique s'est versée dans le mimétisme français, dans le cadre de la lutte contre le Coronavirus. Le Ministre des finances, dans sa lutte contre la pandémie du siècle, pris, dans le

15 CMS Francis LEFEBVRE-Avocats, TVA : Quel traitement pour les dons faits par les entreprises? Conséquences fiscales des dons liés au Covid? 28 mai 2020, inwww, cms.law.fra.publication, CMS. Francis Lefebvre, Consulté le 19/12/2020, à 14h30.

16 CMS Francis LEFEBVRE-Avocats, TVA : Quel traitement pour les dons faits par les entreprises? Cons2quences fiscales des dons liés au Covid? 28 mai 2020, incms.law.fra.publication, CMS. Francis Lefebvre, Consulté le 19/12/2020, à 14h30.

17 Bulletin Officiel des Finances Publiques-Impôts- Le site des commentaires officiels des dispositions fiscales de la direction Générale des Finances publiques, Rescrit-TVA-Dispense de régularisation de la TVA relative aux dons de biens consentis aux établissements sociaux et médico-sociaux qui accueillent des personnes âgées, des personnes handicapées ou atteintes des pathologies chroniques, aux professionnels de la Santé, aux services de l'Etat et des collectivités territoriales, durât la période de l'état d'urgence sanitaire, In Htps://bofip.impôts.gouv.fr, le 20/12/2020., 15 h06.

18 SFP Finances, Administration générale de la Fiscalité, Taxe sur la valeur ajoutée-Impôts des personnes physiques-Impôts de société, Circulaire 2020/C/46 concernant les dons de biens à certains établissements et les dons en nature, in www2.deloitte.com, consulté le 20/12/2020, à $13 \mathrm{~h} 54$. 
même sens que le Ministre de finances de la R.D.Congo, compte tenu de l'urgence et de la gravité de la situation pour la population, des mesures fiscales temporaires d'exception et relatives à la TVA, à l'impôt sur le Société et aux dons en nature donnés aux établissements déterminés par la circulaire, et lieu que ces mesures soient au-delà de l'état d'urgence, et même à chaque exercice fiscal.

Partant du principe : « lorsqu'une entreprise assujetti à la TVA transmet à titre gratuit ses biens meubles corporels, matériels, non vendus, achetés ou fabriqués par elle, cette opération serait une livraison à soi-même ", sans que ce soit dans l'intérêt personnel de l'entreprise, assimilée à une livraison à titre onéreux et ne peut ouvrir droit au mécanisme de déductibilité, sinon il y aura une régularisation ${ }^{19}$.

Le gouvernement belge a constaté que des nombreux sociétés commerciales ont été confronté à cette question de régularisation de la TVA et d'éventuelle fraude à la tva et constituerait un acte anormal de gestion, alors qu'elles étaient en mesure d'apporter aux organismes et professionnels de santé et aux établissements hospitaliers et des organismes de protection des personnes âgées, qui étaient pénuries, des biens liés au covid-19, des appareils respiratoires, des lits et des campements, dans le cadre de la solidarité nationale à la lutte contre la pandémie coronavirus.

Dans le cadre de l'état d'urgence, la TVA pour les livraisons gratuite par les entreprises des biens meubles corporels liés au Covid- $19^{20}$ à des tiers ${ }^{21}$, en vue de leur utilisation dans le cadre de leur activité normale, a été supprimée. Le service des décisions anticipées belge a accepté que l'employeur octroie une intervention non imposable et forfaitaire pour les travailleurs qui a été autorisé au télétravai1 22 . Contrairement à la «directive », la justification des dons est également assouplie : le bénéficiaire du don est dispensé de délivrer l'attestation mentionnée ci-dessus, seulement, l' « entreprise donatrice » conservera, à l'appui de sa comptabilité, les écritures comptables et informations nécessaires permettant d'identifier la date du don, le bénéficiaire du don, la nature et les quantités de biens donnés.

19 SFP Finances, Administration générale de la Fiscalité, Taxe sur la valeur ajoutée-Impôts des personnes physiques-Impôts de société, Circulaire 2020/C/46 concernant les dons de biens à certains établissements et les dons en nature, in www2.deloitte.com, consulté le 20/12/2020, à 13h54.

20 De matériels sanitaires, masques et d'écrans faciaux, gels et solutions hydro alcooliques, tenues de protection contre Covid et les appareils respirateurs, les tenues professionnels contre Covid-19.

21 Au profit des organismes bénéficiaires suivants : les établissements de santé, les établissements et services d'accueil de personnes âgées ou handicapées, les professionnels de santé, les services de l'Etat et les collectivités territoriales.

22 Francis LEVEBVRE, Travail à domicile à cause du COVID-19 : un remboursement des frais propres à l'employeur est-il possible?, in https://www.agoria.be/fr/Travail-a-domicile-a-cause-duCOVID-19-un remboursement-des-frais-propres-q-1-employeur-est-il-possible, 19 mars 2020. 
III. Absence de la loi fiscale et de l'instruction administrative interprétative des articles 13, in fine 41,7 de l'Ordonnance-loi sur la TVA : Risque fiscal de droit de rappel pour fraude à la loi

Bien qu'en matière de la TVA, il y a absence d'une disposition légale qui pourrait permettre aux entreprises commerciale ou industrielle de participer à l'effort de solidarité nationale dans la lutte contre la pandémie de coronavirus et rester non imposable à la livraison des biens liés au covid-19 à titre gratuit qui, du reste est assimilée à la livraison pour soi-même dite à l'autoconsommation des biens affectés à l'objet social de l'entreprise.

Cette lacune juridique devrait être comblée pour que le droit soit en adéquation avec le fait. Dans ce contexte, il serait nécessaire à l'instar de la loi de finances n²0/020 du 28/12/2020 pour l'exercice 2021 prévoyant la déduction au résultat comptable des dons et contributions apportés par les entreprises au Fonds de soutien à la riposte contre le covid-19, comme il est dit dans son article 30. Autrement, il faut prendre des mécanismes administratifs palliatifs, notamment une instruction administrative interprétative des articles 13 , in fine et 41,7 de la loi sur la TVA, le premier relatif à l'exonération, le second à l'exclusion au mécanisme de déductibilité.

\section{Regards critiques sur la législation congolaise}

Loin de tout mimétisme, la R.D. Congo, contrairement à la France et à la Belgique, devraient prendre des mesures fiscales pérennes et adaptées à des situations des entreprises congolaises et aux besoins de la société congolaise, au lieu de passer à des copies servis des modèles français et belge. Le Ministre ayant les finances dans ses attributions, devrait prendre des mesures fiscales d'exception que ce soit avant, pendant et après l'état d'urgence et sans détermination du bénéficiaire de ces biens liés au coronavirus, qu'il soit organismes public ou privé, et cela pour des raisons de coopération et au soutien à l'action gouvernementale, qui se veut être une action intégrale de riposter contre le coronavirus et de sécuriser la santé publique des congolais, au lieu de se passer comme un bon élève de l'Organisation Mondiale de la Santé.

Ce profil de lacune dans la loi sur la TVA en question de l'exonération des biens liés au covid-19, appartenant à la société mais gratuitement livrés par elle, aux tiers-bénéficiaires, organismes professionnels médico-sanitaire, public ou privé, oblige une reforme, dans les jours à venir. Car, s'il a été admis la déduction sur le résultat comptable, des dons et contributions apportés par les entreprises au Fonds de soutien à la riposte contre la pandémie du coronavirus pour l'exercice comptable 2020, le rapprochement est possible pour son exonération à la TVA à la consommation, sinon nous aurons des lois fiscales à deux vitesses.

Avec la maxime : le «fait qui précède le droit », l'instruction administration formellement admise prend forme et, la loi se dissèque! ». 


\section{Perspectives d'avenir et propositions}

Considérant que le covid619 est une pandémie du siècle dont la gouvernance redevable des droits et libertés fondamentaux des citoyens-contribuables au Trésor public oblige respects et garantis, au risque de tuer l'assiette fiscale, par la disparation des contribuables personnes physique et/ou morale, nous demandons aux gouvernants d'avoir une vision plus clairvoyante de la question, au quotidien et non, au passé des faits.

Pour cela, des mesures économiques et fiscales doivent être prises, surtout à l'ère où, le gouvernement actuelle se veut être plus démocratique et au service du peuple, dont la santé publique n'a pas de prix à payer.

Le gouvernant doit devoir, dans le cadre de la protection des citoyens et de l'assiette fiscale, payer un prix fiscal, en prenant des mesures pérennes d'exonérations des biens liés au covid-19 et volontairement remis aux organismes public et/ou privé, par des entreprises en place au Congo, pour autant que ces organismes de santé se trouvent en difficultés de se procurer des biens permettant d'accueillir et de soigner des malades de coronavirus, surtout dans des pays peuples, pauvres et très endettés comme la R.D. du Congo.

Nous préconisons, dans le cadre de combler cette insuffisance logique(légale) face au besoin de la Société congolaise, de voir le gouvernant mettre le peuple à l'abri de cette pandémie, de prendre de mesures gouvernementales de grande envergure, notamment en matière fiscale, à l'instar de la loi de finances $n^{\circ}$ 20/020 du 28/12/2020 pour l'exercice 2021 qui a prévu en son article 30 : “Article 46 bis : "les dons et contributions apportés par les entreprises au Fonds de soutien à la riposte contre la pandémie du coronavirus au cours de l'exercice comptable 2020 soient déductibles du résultat imposable à l'impôt sur les bénéfices et profits, à condition que ces dépenses soient justifiées par les pièces comptables" 23 . Et qu'en matière de la TVA, de prendre une loi de finances reformant l'article 13, in fine et 41,7 de la loi sur la TVA, le premier en exonérant l'importation et la consommation des biens liés au covid-19 livrés gratuitement par les entreprises aux organismes hospitaliers, sanitaires et sociaux public ou privé, au taux zéro de la TVA et, au second article, de ne pas exclure le mécanisme de déductibilité, la livaison à titre gratuit des biens liés au covid-19 à des tiers bénéficiaires précités, et ne point prévoir quant à ce, le mécanisme de régulalation. Sur ce, la TVA declarée et payée en amont, constituerait un crédit TVA à valoir au crédit du compte courant du contribuable pour servir au paiement d'autres impôts et droits dûs au Trésor public.

Dans la même logique de reforme, nous proposons que la loi de finances à venir, ou rectificative de la loi de finances 2021 puisse prévoir le mécanisme des Accords Préalables Fiscaux prises sous formes des décisions fiscales anticipées, avant l'accomplissement du fait générateur de la TVA à l'intérieur, savoir la livraison dite à soi même ou l'autoconsommation de l'entreprise de biens affectés à l'exploitation, mais qui pour des raisons de solida-

23 Loi de finances $n^{\circ} 20 / 020$ du 28/12/2020 pour l'exercice 202, in JORDC, $n^{\circ}$ spécial, 62 ${ }^{\circ}$ année, Ière Partie, Kinshasa, 8 janvier 2021, p. 19. 
rité nationale et de soutien au gouvernement à la riposte de covid-19, apportes ces biens aux organismes publics ou privés, hospitaliers ou sanitaires, en besoin.

Ces entreprises doivent passer avec l'administration fiscale des Accords préalables fiscaux » afin de se voir protéger contre le droit de rappeler les impôts et de participer sans risque fiscal à l'effort mondial de lutte contre le coronavirus,

Un autre palliatif serait les prises par l'administration fiscale des instructions administratives ou circulaires interprétatives des articles 13 , in fine et 41,7 de la loi sur la TVA, toute en déterminant que ces entreprises qui participent au Fonds de soutien, à la livraison gratuit des biens, non pour des besoins publicitaires qui seraient de promotion et d'exploitation commerciale, mais plus pour des besoins de lutter contre la pandémie de covid-19, doivent être exonérées de la TVA à l'importation et à l'intérieur (l'entreprise se comportant comme consommateur final) et ne pourrait être exclu de mécanisme de déductibilité, et leur situation fiscale ne pourrait être régulariser, telle est le sens de l'article 39 apportant une garantie au contribuable contre le changement de la doctrine administrative.

\section{Conclusion}

«Aider son prochain est une maxime qui n'est pas du goût du fisc », surtout quand elle est pratiquée par une entreprise dont la mission est de réaliser le bénéfice et de profiter de son économie, et non d'exercer la charité24.

Le contour définitionnel et conceptuel du régime fiscal de la livraison à titre gratuit des biens de l'entreprise à des tiers-bénéficiaires que sont les organismes public et privé, d'ordre sanitaire et hospitalier, à l'ère de la pandémie et des entreprises commerciales et industrielles qui évoluent dans un environnement, économiquement, moins recommandables obligent le gouvernement à prendre des décisions redevables des droits fondamentaux des citoyens. S'il recours au capital privé pour participer à la lutte contre la pandémie, ce qui n'est pas la mission de l'entreprise, il doit devoir sur le plan économique que fiscal prendre des mesures des réformes protectrices des entreprises et les sécurisant contre les risques fiscaux de redressements d'assiette et des pénalités de recouvrement.

C'est dans ce cadre que nous estimons que ces trois mécanismes s'avèrent importants, hormis la loi de finances $n^{\circ} 20 / 020$ du 28/12/2020 pour l'exercice 2021, du 8 janvier 2021, qui déduit dans le résultat imposable à l'impôt sur les bénéfices et profits, les dons et contributions apportés par les entreprises au Fond de soutien à la riposte contre la pandémie du coronavirus au cours de l'exercice comptable 2020. Et, proposons qu'à chaque exercice comptable cette disposition, aménagée pour l'exercice, soit reconduit. Ces trois mécanismes précités sont :

- Qu'en matière de la TVA, de prendre une loi de finances reformant l'article 13, in fine et 41,7 de la loi sur la TVA, le premier en exonérant l'importation et la consommation des biens liés au covid-19 livrés gratuitement par les entreprises aux organismes hospitaliers, sanitaires et sociaux public ou privé, au taux zéro de la TVA et, au second article à ne pas exclure cette opération au de la livraison gratuite des biens liés au cronavirus

24 MAURICE COZIAN, Précis de fiscalité des entreprises, $28^{\text {mème }}$ édition, Paris, 2004, p.238. 
aux tiers-bénéficiaires au mécanisme de déductibilité, et ne point prévoir quant à ce, la régulation de la TVA;

- Que de lege ferenda, qu'une loi de finances rectificative de la loi de finances 2021 soit prise, et prévoir le mécanisme des Accords Préalables Fiscaux prises, entre les deux partenaires fiscaux, le fisc et le contribuable, et coulés sous formes des décisions fiscales anticipées, bien avant que l'entreprise procède à la livraison à titre gratuit des biens liés au covid-19 aux organismes public et privé, d'ordre sanitaire et hospitalier, puis des hommes de vieillards et des personnes vulnérables, alors que ces biens sont censés être commercialisés, et puis;

- Que l'administration fiscale puisse prendre des instructions administratives ou circulaires interprétatives des articles 13 , in fine et 41,7 de la loi sur la TVA, en déterminant que ces entreprises qui participent à la livraison gratuite des biens pour des besoins de lutter contre la pandémie de covid-19, soient exonérées de la TVA à l'importation et à la consommation, et que les TVA déclarées et payées en amont puisse constituer, en vertu du mécanisme de déductibilité, un crédit TVA à inscrire au crédit du compte courent du contribuable et à valoir sur les paiements des autres impôts et droits dûs au Trésor, remboursement possible après la perte de la qualité d'assujetti.

\section{BIBLIOGRAPHIE}

\section{A. OUVRAGES}

I. Aubry GILDAS et Cie, Fiscal, $2^{\text {ème }}$ édition, Paris, 2017.

II. Francis LEFEBVRE, Fiscal, Paris, 2012.

III. Gildas AUBRIL et Cie, Fiscal, Allemagne, 2017

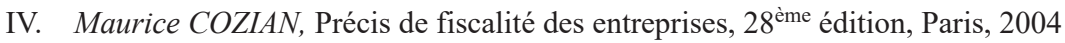

V. Maurice COZIAN, Précis de fiscalité des entreprises, 34è édition, Paris, 2010.

VI. François PILLET et Eugène BANGOURA, Guide pratique de la procédure dans le contentieux fiscal, 3è édition, Paris, 2003.

\section{B. TEXTES LEGAUX ET REGLEMENTAIRES}

I. Constitutions de la République Démocratique du Congo modifiée par la loi n 11/002 du 20 janvier 2011 portant révision de certains articles de la Constitution de la République Démocratique du Congo du 18 février 2006, in JORDC, $52^{\text {ème }}$ Année, Numéro Spécial, Kinshasa, 5 février 2011.

II. Loi $\mathrm{n}^{\circ}$ 004/2003 du 13 mars 2003 portant réforme des procédures fiscales, telle que modifiée et complétée à ce jours, in Code Général des Impôts, Kinshasa, 2007.

III. Ordonnance-loi $n^{\circ} 13 / 007$ du 23 février 2013 modifiant et complétant certaines dispositions de l'ordonnance-loi $\mathrm{n}^{\circ} 10 / 001$ du 20/08/2010 portant institution de la Taxe sur la Valeur Ajoutée, in Journal officiel - Numéro Spécial, Kinshasa - 2 mars 2013. 
IV. Loi des finances $n^{\circ} 20 / 020$ du 28/12/2020 pour l'exercice 2021, in JORDC, $62^{\circ}$ année, Numéro Spécial, Kinshasa - 8 janvier 2021.

V. Décret $\mathrm{n}^{\circ}$ 011/42 du 22 Novembre 2011 fixant les mesures d'exécution de l'Ordonnance-Loi $\mathrm{n}^{\circ}$ 10/011 du 20 Août 2010 portant institution de la TVA, in www. leganet.cd.Droitcongolais.be, 2011.

\section{SITE INTERNET}

I. CMS Francis LEFEBVRE-Avocats, TVA : Quel traitement pour les dons faits par les entreprises? Conséquences fiscales des dons liés au Covid, 28 mai 2020, in www, cms.law.fra.publication, CMS. Francis Lefebvre.

II. Francis LEVEBVRE, Travail à domicile à cause du COVID-19 : un remboursement des frais propres à l'employeur est-il possible?, in https://www.agoria.be/fr/Travail-adomicile-a-cause-du-COVID-19- un remboursement-des-frais-propres-q-1-employeurest-il-possible.

III. SFP Finances, Administration générale de la Fiscalité, Taxe sur la valeur ajoutée-Impôts des personnes physiques-Impôts de société, Circulaire 2020/C/46 concernant les dons de biens à certains établissements et les dons en nature, in $\mathrm{w}$

IV. ww2.deloitte.com

\section{DOCUMENTS OFFICIELS}

I. Bulletin Officiel des Finances Publiques-Impôts- Le site des commentaires officiels des dispositions fiscales de la direction Générale des Finances publiques, RescritTVA-Dispense de régularisation de la TVA relative aux dons de biens consentis aux établissements sociaux et médico-sociaux qui accueillent des personnes âgées, des personnes handicapées ou atteintes des pathologies chroniques, aux professionnels de la Santé, aux services de l'Etat et des collectivités territoriales, durât la période de l'état d'urgence sanitaire, In Htps://bofip.impôts.gouv.fr

II. Lettre $n^{\circ} C A B / P M / D I R C A B / G N / 2020 / 0686$ du 07Avril 2020 du Premier Ministre au Ministre des Finances de la R.D.Congo, inédit.

III. Lettre $n^{\circ} C A B / M I N / F I N A N C E S / 2020 / 1092$ du Ministre des Finances adressée au Directeur Général des Impôts, inédit.

IV. République Démocratique du Congo, Ministère des Finances, Code des Impôts, Kinshasa, 2014. 